\title{
COMBINATORIAL RESULTS FOR CERTAIN SEMIGROUPS OF ORDER-DECREASING PARTIAL ISOMETRIES OF A FINITE CHAIN
}

\section{F. AL-KHAROUSI ${ }^{1}$, R. KEHINDE ${ }^{2}$ and A. UMAR ${ }^{3}$}

1Department of Mathematics and Statistics

Sultan Qaboos University

Al-Khod, PC 123

Oman

e-mail: fatma9@squ.edu.om

2Department of Mathematics and Statistics

Bowen University

P. M. B. 284, Iwo

Osun State

Nigeria

e-mail: kennyrot2000@yahoo.com

${ }^{3}$ Department of Mathematics

Petroleum Institute

Khalifa University of Science and Technology

Sas Nakhl, P. O. Box 2533

Abu Dhabi

U. A. E.

e-mail: aumar@pi.ac.ae

2010 Mathematics Subject Classification: 20M18, 20M20, 05A10, 05A15.

Keywords and phrases: partial one-one transformation, partial isometries, height, right (left) waist, right (left) shoulder and fix of a transformation, idempotents and nilpotents.

Financial support from Sultan Qaboos University Internal Grant: IG/SCI/DOMS/13/06 is gratefully acknowledged.

Received December 1, 2017

(C) 2018 Scientific Advances Publishers 


\begin{abstract}
Let $\mathcal{I}_{n}$ be the symmetric inverse semigroup on $X_{n}=\{1,2, \ldots, n\}$ and let $\mathcal{D D P}_{n}$ and $\mathcal{O D D P}_{n}$ be its subsemigroups of order-decreasing partial isometries and of order-preserving and order-decreasing partial isometries of $X_{n}$, respectively. In this paper, we investigate the cardinalities of some equivalences on $\mathcal{D D P}_{n}$ and $\mathcal{O D D P}_{n}$, which lead naturally to obtaining the order of the semigroups.
\end{abstract}

\title{
1. Introduction
}

Let $X_{n}=\{1,2, \ldots, n\}$ and $\mathcal{I}_{n}$ be the partial one-to-one transformation semigroup on $X_{n}$ under composition of mappings. Then $\mathcal{I}_{n}$ is an inverse semigroup (that is, for all $\alpha \in \mathcal{I}_{n}$ there exists a unique $\alpha^{\prime} \in \mathcal{I}_{n}$ such that $\alpha=\alpha \alpha^{\prime} \alpha$ and $\left.\alpha^{\prime}=\alpha^{\prime} \alpha \alpha^{\prime}\right)$. The importance of $\mathcal{I}_{n}$ (more commonly known as the symmetric inverse semigroup or monoid) to inverse semigroup theory may be likened to that of the symmetric group $\mathcal{S}_{n}$ to group theory. Every finite inverse semigroup $S$ is embeddable in $\mathcal{I}_{n}$, the analogue of Cayley's theorem for finite groups. Thus, just as the study of symmetric, alternating and dihedral groups has made a significant contribution to group theory, so has the study of various subsemigroups of $\mathcal{I}_{n}$, see, for example, $[3,5,6,10,14,19,20]$.

A transformation $\alpha \in \mathcal{I}_{n}$ is said to be order-preserving (orderreversing) if $(\forall x, y \in \operatorname{Dom} \alpha) x \leq y \Rightarrow x \alpha \leq y \alpha(x \alpha \geq y \alpha)$ and, an isometry (or distance-preserving) if $(\forall x, y \in \operatorname{Dom} \alpha)|x-y|=|x \alpha-y \alpha|$. We shall denote by $\mathcal{D P}_{n}$ and $\mathcal{O D P}{ }_{n}$, the semigroups of partial isometries and of order-preserving partial isometries of an $n$-chain, respectively. Eventhough semigroups of partial isometries on more restrictive but richer mathematical structures have been studied by Wallen [21], and Bracci and Picasso [4] the study of the corresponding semigroups on chains was only initiated recently by Al-Kharousi et al. [1, 2]. A little while later, Kehinde et al. [13] studied $\mathcal{D D P}_{n}$ and $\mathcal{O D D P}_{n}$, the orderdecreasing analogues of $\mathcal{D P}{ }_{n}$ and $\mathcal{O D P}{ }_{n}$, respectively. 
Analogous to Al-Kharousi et al. [2], this paper investigates the combinatorial properties of $\mathcal{D D P}{ }_{n}$ and $\mathcal{O D D P}_{n}$, thereby complementing the results in Kehinde et al. [13] which dealt mainly with the algebraic and rank properties of these semigroups. In this section we introduce basic definitions and terminology as well as quote some elementary results from Section 1 of Al-Kharousi et al. [1] and Kehinde et al. [13] that will be needed in this paper. In Section 2, we obtain the cardinalities of two equivalences defined on $\mathcal{O D D P}_{n}$ and $\mathcal{D D P}{ }_{n}$. These equivalences lead to formulae for the orders of $\mathcal{O D D P}_{n}$ and $\mathcal{D D P}{ }_{n}$ as well as new triangles of numbers that were as a result of this work recently recorded in [18].

For standard concepts in semigroup and symmetric inverse semigroup theory, see, for example, $[12,16]$. In particular $E(S)$ denotes the set of idempotents of $S$. Let

$$
\mathcal{D D P}_{n}=\left\{\alpha \in \mathcal{D P}_{n}:(\forall x \in \operatorname{Dom} \alpha) x \alpha \leq x\right\} .
$$

be the subsemigroup of $\mathcal{I}_{n}$ consisting of all order-decreasing partial isometries of $X_{n}$. Also let

$$
\mathcal{O D D P}_{n}=\left\{\alpha \in \mathcal{D D P}_{n}:(\forall x, y \in \operatorname{Dom} \alpha) x \leq y \Rightarrow x \alpha \leq y \alpha\right\},
$$

be the subsemigroup of $\mathcal{D D P}_{n}$ consisting of all order-preserving and order-decreasing partial isometries of $X_{n}$. Then we have the following result.

Lemma 1.1. $\mathcal{D D P}_{n}$ and $\mathcal{O D D P}{ }_{n}$ are subsemigroups of $\mathcal{I}_{n}$.

Remark 1.2. $\mathcal{D D P}_{n}=\mathcal{D P}_{n} \cap \mathcal{I}_{n}^{-} \quad$ and $\quad \mathcal{O D D P}_{n}=\mathcal{O D P}_{n} \cap \mathcal{I}_{n}^{-}$, where $\mathcal{I}_{n}^{-}$is the semigroup of partial one-to-one order-decreasing transformations of $X_{n}$ [19].

Next, let $\alpha$ be an arbitrary element in $\mathcal{I}_{n}$. The height or rank of $\alpha$ is $h(\alpha)=|\operatorname{Im} \alpha|$, the right $[$ left $]$ waist of $\alpha$ is $w^{+}(\alpha)=\max (\operatorname{Im} \alpha)\left[w^{-}(\alpha)\right.$ 
$=\min (\operatorname{Im} \alpha)]$, the right [left] shoulder of $\alpha$ is $\varpi^{+}(\alpha)=\max (\operatorname{Dom} \alpha)$ $[\varpi(\alpha)=\min (\operatorname{Dom} \alpha)]$, and fix of $\alpha$ is denoted by $f(\alpha)$, and defined by $f(\alpha)=|F(\alpha)|$, where

$$
F(\alpha)=\left\{x \in X_{n}: x \alpha=x\right\}
$$

Next we quote some parts of ([1], Lemma 1.2) that will be needed as well as state some additional observations that will help us understand more the cycle structure of order-decreasing partial isometries.

Lemma 1.3. Let $\alpha \in \mathcal{D P}{ }_{n}$. Then we have the following:

(a) The map $\alpha$ is either order-preserving or order-reversing. Equivalently, $\alpha$ is either a translation or a reflection.

(b) If $f(\alpha)=p>1$, then $f(\alpha)=h(\alpha)$. Equivalently, if $f(\alpha)>1$, then $\alpha$ is a partial identity.

(c) If $\alpha$ is order-preserving and $f(\alpha) \geq 1$, then $\alpha$ is a partial identity.

(d) If $\alpha$ is order-preserving then it is either strictly order-decreasing $(x \alpha<x$ for all $x$ in Dom $\alpha)$ or strictly order-increasing $(x \alpha>x$ for all $x$ in Dom $\alpha$ ) or a partial identity.

(e) If $F(\alpha)=\{i\}($ for $1 \leq i \leq n)$, then for all $x \in$ Dom $\alpha$ we have that $x+x \alpha=2 i$.

(f) If $\alpha$ is order-decreasing and $i \in F(\alpha)(1 \leq i \leq n)$, then for all $x \in$ Dom $\alpha$ such that $x<i$ we have $x \alpha=x$.

(g) If $\alpha$ is order-decreasing and $F(\alpha)=\{i\}$, then Dom $\alpha \subseteq\{i, i+1, \ldots, n\}$.

\section{Combinatorial Results}

For a nice survey article concerning combinatorial problems in the symmetric inverse semigroup and some of its subsemigroups, we refer the reader to Umar [20]. 
As in Umar [20], for natural numbers $n \geq p \geq m \geq 0$ and $n \geq i \geq 0$, we define

$$
\begin{gathered}
F\left(n ; p_{i}\right)=|\{\alpha \in S: h(\alpha)=|\operatorname{Im} \alpha|=i\}|, \\
F\left(n ; m_{i}\right)=|\{\alpha \in S: f(\alpha)=i\}|,
\end{gathered}
$$

where $S$ is any subsemigroup of $\mathcal{I}_{n}$. From ([2], Proposition 2.4), we have

Theorem 2.1. Let $S=\mathcal{O D P}_{n}$. Then $F(n ; p)=\frac{(2 n-p+1)}{p+1}\left(\begin{array}{l}n \\ p\end{array}\right)$, where $n \geq p \geq 1$.

We now have

Proposition 2.2. Let $S=\mathcal{O D D P}_{n}$. Then $F(n ; p)=\left(\begin{array}{c}n+1 \\ p+1\end{array}\right)$, where $n \geq p \geq 1$

Proof. By virtue of Lemma 1.3 (d) and Theorem 2.1, we see that

$$
\begin{aligned}
F(n ; p) & =\frac{1}{2}\left[\frac{2 n-p+1}{p+1}\left(\begin{array}{l}
n \\
p
\end{array}\right)-\left(\begin{array}{l}
n \\
p
\end{array}\right)\right]+\left(\begin{array}{l}
n \\
p
\end{array}\right) \\
& =\frac{1}{2}\left[\frac{2(n-p)}{p+1}\left(\begin{array}{l}
n \\
p
\end{array}\right)\right]+\left(\begin{array}{l}
n \\
p
\end{array}\right) \\
& =\frac{n-p}{p+1}\left(\begin{array}{l}
n \\
p
\end{array}\right)+\left(\begin{array}{l}
n \\
p
\end{array}\right)=\left(\begin{array}{c}
n \\
p+1
\end{array}\right)+\left(\begin{array}{l}
n \\
p
\end{array}\right)=\left(\begin{array}{l}
n+1 \\
p+1
\end{array}\right) .
\end{aligned}
$$

The proof of the next lemma is routine using Proposition 2.2.

Lemma 2.3. Let $S=\mathcal{O D D P}_{n}$. Then $F(n ; p)=F(n-1 ; p-1)+F$ $(n-1 ; p)$, for all $n \geq p \geq 2$.

Theorem 2.4. $\left|\mathcal{O D D P}_{n}\right|=2^{n+1}-(n+1)$. 
Proof. It is enough to observe that $\left|\mathcal{O D D P}_{n}\right|=\sum_{p=0}^{n} F(n ; p)$.

Lemma 2.5. Let $S=\mathcal{O D D P}_{n}$. Then $F(n ; m)=\left(\begin{array}{l}n \\ m\end{array}\right)$, for all $n \geq m \geq 1$.

Proof. It follows directly from Lemma 1.3 (b), (c) and the fact that all idempotents are necessarily order-decreasing.

Proposition 2.6. Let $U_{n}=\left\{\alpha \in \mathcal{O D D P}_{n}: f(\alpha)=0\right\}$. Then $\left|U_{n}\right|=$ $\left|\mathcal{O D D} \mathcal{P}_{n-1}\right|$.

Proof. The proof is similar to that of ([19], Theorem 4.3).

Remark 2.7. The triangles of numbers $F(n ; p)$ and $F(n ; m)$, have as a result of this work appeared in Sloane [18] as [A184049] and [A184050], respectively.

Now we turn our attention to counting order-reversing partial isometries. First recall from ([13], Section 3.2 (c)) that order-decreasing and order-reversing partial isometries exist only for heights less than or equal to $n / 2$. We now have

Lemma 2.8. Let $S=\mathcal{D D P}_{n}^{*}$ be the set of order-reversing partial isometries of $X_{n}$. Then $F\left(n ; p_{0}\right)=1$ and $F\left(n ; p_{1}\right)=\left(\begin{array}{c}n+1 \\ 2\end{array}\right)$, for all $n \geq 1$.

Proof. These follow from the simple observation that

$$
\left\{\alpha \in \mathcal{O D D P}_{n}: h(\alpha)=0 \text { or } 1\right\}=\left\{\alpha \in \mathcal{D D P}_{n}^{*}: h(\alpha)=0 \text { or } 1\right\}
$$

and Proposition 2.2 .

Lemma 2.9. Let $\alpha \in \mathcal{D D P}_{n}^{*}$. Then for all $p \geq 1$ we have

$$
F(2 p+1, p+1)=1 \text { and } F(2 p, p)=3 .
$$


Proof. (i) By Lemma 1.3 (f), (g), we see that $i \in\{0,1, \ldots, p\},\left(\begin{array}{l}p+1+i \\ p+1-i\end{array}\right)$ is the unique order-reversing isometry of height $p+1$; and (ii) for $i \in\{0,1, \ldots, p-1\},\left(\begin{array}{c}p+i \\ p-i\end{array}\right),\left(\begin{array}{c}p+1+i \\ p-i\end{array}\right)$, and $\left(\begin{array}{c}p+1+i \\ p+1-i\end{array}\right)$ are the only order-reversing isometries of height $p$.

The following technical lemma will be useful later.

Lemma 2.10. Let $\alpha \in \mathcal{D D P}_{n}^{*}$. Suppose $\varpi^{+}(\alpha)-r \in \operatorname{Dom} \alpha$ and $\varpi^{+}(\alpha)-s \notin$ Dom $\alpha$ for all $1 \leq s<r$. Then $\varpi(\alpha)>r$.

Proof. By order-reversing we see that $\left(\varpi^{+}(\alpha)\right) \alpha=w^{-}(\alpha)$ and $(\varpi(\alpha))$ $\alpha=w^{+}(\alpha)$. Thus $\varpi^{+}(\alpha)-r \geq \varpi(\alpha) \Rightarrow \varpi^{+}(\alpha)-\varpi(\alpha) \geq r$. So by isometry we have $w^{+}(\alpha)-w^{-}(\alpha)=\varpi^{+}(\alpha)-\varpi(\alpha) \geq r \Rightarrow w^{+}(\alpha) \geq w^{-}(\alpha)+r \Rightarrow w^{+}$ $(\alpha)>r \Rightarrow \varpi(\alpha)>r$, as required.

Lemma 2.11. Let $S=\mathcal{D D P}_{n}^{*}$. Then $F(n ; p)=F(n-2 ; p-1)+$ $F(n-2 ; p)$, for all $n \geq p \geq 2$.

Proof. Let $\alpha \in \mathcal{D D P}_{n}^{*}$ and $h(\alpha)=p$. Define $A=\left\{\alpha \in \mathcal{D D P}_{n-2}^{*}\right.$ : $h(\alpha)=p\}$ and $B=\left\{\alpha \in \mathcal{D D P P}_{n-2}^{*}: h(\alpha)=p-1\right\}$. Clearly, $A \cap B=\emptyset$. Define a map $\theta:\left\{\alpha \in \mathcal{D D P}_{n}^{*}: h(\alpha)=p\right\} \rightarrow A \cup B$ by $(\alpha) \theta=\alpha^{\prime}$, where

(i) $x \alpha^{\prime}=x \alpha(x \in \operatorname{Dom} \alpha)$, if $\alpha \in A$. It is clear that $\alpha^{\prime}$ is an orderdecreasing isometry and $h(\alpha)=p$;

(ii) if $\{n-1, n\} \subseteq$ Dom $\alpha\}$ and $\alpha \in B$, let Dom $\alpha^{\prime}=\{x-1: x \in$ Dom $\alpha$ and $x<n\}$ and $(x-1) \alpha^{\prime}=x \alpha-1 \leq x-1$ and so $\alpha^{\prime}$ is orderdecreasing and $h(\alpha)=p-1$; 
(iii) if $\{n-2, n-1\} \subseteq$ Dom $\alpha\}$ and $\alpha \in B$, let Dom $\alpha^{\prime}=\{x-1: x \in$ Dom $\alpha$ and $x<n-1\}$ and $(x-1) \alpha^{\prime}=x \alpha-1 \leq x-1$ and so $\alpha^{\prime}$ is orderdecreasing and $h(\alpha)=p-1$;

(iv) otherwise, if $\alpha \in B$, let Dom $\alpha^{\prime}=\{x-r: x \in$ Dom $\alpha$ and $\left.x<\varpi^{+}(\alpha)\right\}$, where $r$ is such that $\varpi^{+}(\alpha)-r \in \operatorname{Dom} \alpha$ and $\varpi^{+}(\alpha)-s \notin$ Dom $\alpha$ for all $1 \leq s<r$. Define $(x-r) \alpha^{\prime}=x \alpha-r \leq x-r$ and so $\alpha^{\prime}$ is order-decreasing and Lemma 2.10 ensures that $h(\alpha)=p-1$.

Moreover, in (ii) and (iii), we have $\left|(x-1) \alpha^{\prime}-(y-1) \alpha^{\prime}\right|=\mid(x \alpha-1)-$ $(y \alpha-1)|=| x \alpha-y \alpha|=| x-y|=|(x-1)-(y-1) \mid$, and in (iv), we have $\left|(x-r) \alpha^{\prime}-(y-r) \alpha^{\prime}\right|=|(x \alpha-r)-(y \alpha-r)|=|x \alpha-y \alpha|=|x-y|=\mid(x-r)-$ $(y-r) \mid$. Hence $\alpha^{\prime}$ is an isometry.

Also observe that in (ii), we have $\varpi^{+}\left(\alpha^{\prime}\right)=n-2$; in (iii) we have $\varpi^{+}\left(\alpha^{\prime}\right)=n-3$; and in (iv) we have $\varpi^{+}\left(\alpha^{\prime}\right)<n-3$. These observations coupled with the definitions of $\alpha^{\prime}$ ensures that $\theta$ is a bijection.

To show that $\theta$ is onto it is enough to note that we can in a symmetric manner define $\theta^{-1}$ from $A \cup B \rightarrow\left\{\alpha \in \mathcal{D D P}_{n}^{*}: h(\alpha)=p\right\}$. This establishes the statement of the lemma.

The next lemma which can be proved by induction, is necessary.

Lemma 2.12. Let $S=\mathcal{D D P}_{n}^{*}$. Then we have the following:

$$
\sum_{i \geq 0}\left(\begin{array}{c}
n-1-2 i \\
2
\end{array}\right)= \begin{cases}\frac{(n+1)(n-1)(2 n-3)}{24}, & \text { if } n \text { is odd } \\
\frac{n(n-2)(2 n+1)}{24}, & \text { if } n \text { is even } .\end{cases}
$$

Lemma 2.13. Let $S=\mathcal{D D P}_{n}^{*}$. Then we have the following:

$$
F\left(n ; p_{2}\right)= \begin{cases}\frac{(n+1)(n-1)(2 n-3)}{24}, & \text { if } n \text { is odd } \\ \frac{n(n-2)(2 n+1)}{24}, & \text { if } n \text { is even. }\end{cases}
$$


Proof. By applying Lemmas 2.8 and 2.11 successively, we get

$$
\begin{aligned}
F\left(n ; p_{2}\right) & =F\left(n-2 ; p_{1}\right)+F\left(n-2 ; p_{2}\right)=F\left(n-2 ; p_{2}\right)+\left(\begin{array}{c}
n-1 \\
2
\end{array}\right) \\
& =F\left(n-4 ; p_{2}\right)+\left(\begin{array}{c}
n-3 \\
2
\end{array}\right)+\left(\begin{array}{c}
n-1 \\
2
\end{array}\right) \\
& =F\left(n-6 ; p_{2}\right)+\left(\begin{array}{c}
n-5 \\
2
\end{array}\right)+\left(\begin{array}{c}
n-3 \\
2
\end{array}\right)+\left(\begin{array}{c}
n-1 \\
2
\end{array}\right) .
\end{aligned}
$$

By iteration the result follows from Lemma 2.12 and the facts that $F\left(2 ; p_{2}\right)=0$ and $F\left(3 ; p_{2}\right)=1=\left(\begin{array}{l}2 \\ 2\end{array}\right)$.

Proposition 2.14. Let $S=\mathcal{D D P}_{n}^{*}$. Then for all $\lfloor(n+1) / 2\rfloor \geq p \geq 1$, we have

$F(n ; p)= \begin{cases}\frac{(n+1)(n-1)(n-3) \cdots(n-2 p+3)(2 n-3 p+3)}{2^{p}(p+1) !}, & \text { if } n \text { is odd } \\ \frac{n(n-2)(n-4) \cdots(n-2 p+2)(2 n-p+3)}{2^{p}(p+1) !}, & \text { if } n \text { is even. }\end{cases}$

Proof (By Induction).

Basis step: $F\left(n ; p_{1}\right)=\left(\begin{array}{c}n+1 \\ 1+1\end{array}\right)=\left(\begin{array}{c}n+1 \\ 2\end{array}\right)$ is true by Lemma 2.8 and the observation made in its proof, while the formula for $F\left(n ; p_{2}\right)$ is true by Lemma 2.13.

Inductive step: Suppose $F(m ; p)$ is true for all $\lfloor(n+1) / 2\rfloor>m \geq p \geq 1$. 
Case 1. If $m$ is odd, consider (using the induction hypothesis)

$$
\begin{aligned}
F(m+2 ; p)= & F(m ; p)+F(m ; p-1) \\
= & \frac{(m+1)(m-1)(m-3) \cdots(m-2 p+3)(2 m-3 p+3)}{2^{p}(p+1) !} \\
& +\frac{(m+1)(m-1)(m-3) \cdots(m-2 p+5)(2 m-3 p+6)}{2^{p-1} p !} \\
= & \frac{(m+3)(m+1)(m-1) \cdots(m-2 p+5)(2 m-3 p+7)}{2^{p}(p+1) !}
\end{aligned}
$$

which is the formula for $F(m+2 ; p)$ when $m$ is odd.

Case 2. If $m$ is even, consider (using the induction hypothesis)

$$
\begin{aligned}
F(m+2 ; p)= & F(m ; p)+F(m ; p-1) \\
= & \frac{m(m-2)(m-4) \cdots(m-2 p+2)(2 m-p+3)}{2^{p}(p+1) !} \\
& +\frac{m(m-2)(m-4) \cdots(m-2 p+4)(2 m-p+4)}{2^{p-1} p !} \\
= & \frac{(m+2) m(m-2) \cdots(m-2 p+4)(2 m-p+7)}{2^{p}(p+1) !},
\end{aligned}
$$

which is the formula for $F(m+2 ; p)$ when $m$ is even.

Proposition 2.15. Let $S=\mathcal{D D P}_{n}^{*}$ and let $b_{n}=\sum_{p \geq 0} F(n ; p)$. Then for $n \geq 0$, we have

(1) $b_{2 n+1}=5 \cdot 2^{n+1}-4 n-8$;

(2) $b_{2 n}=7 \cdot 2^{n}-4 n-6$.

Proof. Apply induction and use the fact that $\left|\mathcal{D D} \mathcal{P}_{n}^{*}\right|=\sum_{p=0}^{n} F(n ; p)$. 
Proposition 2.16. Let $S=\mathcal{D D P}_{n}$. Then

(1) if $n$ is odd and $(n+1) / 2 \geq p \geq 2$

$F(n ; p)=\frac{(n+1)(n-1)(n-3) \cdots(n-2 p+3)(2 n-3 p+3)}{2^{p}(p+1) !}+\left(\begin{array}{l}n+1 \\ p+1\end{array}\right)$

(2) if $n$ is even and $n / 2 \geq p \geq 2$

$$
F(n ; p)=\frac{n(n-2)(n-4) \cdots(n-2 p+2)(2 n-p+3)}{2^{p}(p+1) !}+\left(\begin{array}{c}
n+1 \\
p+1
\end{array}\right)
$$

(3) if $\lfloor(n+1) / 2\rfloor<p, F(n ; p)=\left(\begin{array}{c}n+1 \\ p+1\end{array}\right)$.

Proof. It follows from Propositions $2.2 \& 2.14$ and Lemmas 1.3 (c) \& 2.8 .

Combining Theorem 2.4, Lemmas 1.3 (a), (c) \& 2.9, Proposition 2.15 and the observation made in the proof of Lemma 2.8 we get the order of $\mathcal{D D P} \mathcal{P}_{n}$ which we record as a theorem below.

Theorem 2.17. Let $\mathcal{D D P}_{n}$. Then for all $n \geq 0$, we have

(1) $\left|\mathcal{D D} \mathcal{P}_{2 n+1}\right|=2^{2 n+2}+5 \cdot 2^{n+1}-\left(2 n^{2}+9 n+12\right)$;

(2) $\left|\mathcal{D D P} \mathcal{P}_{2 n}\right|=2^{2 n+1}+7 \cdot 2^{n}-\left(2 n^{2}+7 n+8\right)$.

Lemma 2.18. Let $S=\mathcal{D D P}_{n}$. Then $F(n ; m)=\left(\begin{array}{l}n \\ m\end{array}\right)$, for all $n \geq m \geq 2$.

Proof. It follows directly from ([13], Lemma 3.18) and the fact that all idempotents are necessarily order-decreasing.

Proposition 2.19. Let $S=\mathcal{D D P}_{n}$. Then $F\left(2 n ; m_{1}\right)=2^{n+1}-2$ and $F\left(2 n-1 ; m_{1}\right)=3 \cdot 2^{n-1}-2$, for all $n \geq 1$. 
Proof. Let $F(\alpha)=\{i\}$. Then by Lemma 1.3 (e), for any $x \in$ Dom $\alpha$ we have $x+x \alpha=2 i$. Thus, by Lemma $1.3(\mathrm{~g})$, there $2 i-2$ possible elements for $\operatorname{Dom} \alpha:(x, x \alpha) \in\{(i, i),(i+1, i-1),(i+2, i-2), \ldots$, $(2 i-1,1)\}$. However, (excluding $(i, i))$ we see that there are $\sum_{j=0}\left(\begin{array}{c}i-1 \\ j\end{array}\right)=2^{i-1}$, possible partial isometries with $F(\alpha)=\{i\}$, where $2 i-1 \leq n \Leftrightarrow i \leq(n+1) / 2$. Moreover, by symmetry, we see that $F(\alpha)=\{i\}$ and $F(\alpha)=\{n-i+1\}$ give rise to equal number of decreasing partial isometries. Note that if $n$ is odd (even) the equation $i=n-i+1$ has one (no) solution. Hence, if $n=2 a-1$ we have

$$
2 \sum_{i=1}^{a-1} 2^{i-1}+2^{a-1}=2\left(2^{a-1}-1\right)+2^{a-1}=3.2^{a-1}-2
$$

decreasing partial isometries with exactly one fixed point; if $n=2 a$ we have

$$
2 \sum_{i=1}^{a} 2^{i-1}=2\left(2^{a}-1\right)=2^{a+1}-2
$$

decreasing partial isometries with exactly one fixed point.

Theorem 2.20. Let $\mathcal{D D P}_{n}$. Then

$$
a_{n}=\left|\mathcal{D D P}_{n}\right|=3 a_{n-1}-2 a_{n-2}-2^{\left\lfloor\frac{n}{2}\right\rfloor}+n+1,
$$

with $a_{0}=1$ and $a_{-1}=0$.

Proof. It follows from Propositions $2.6 \& 2.19$, Lemma 2.18 and the fact that $\left|\mathcal{D D P}_{n}\right|=\sum_{m=0}^{n} F(n ; m)$.

Remark 2.21. The triangle of numbers $F(n ; m)$ and sequence $\left|\mathcal{D D P} \mathcal{P}_{n}\right|$ have as a result of this work appeared in Sloane [18] as [A184051] and [A184052], respectively. However, the triangles of 
numbers $F(n ; p)$ for $\mathcal{D D P}_{n}$ and $\mathcal{D D P}^{*}{ }_{n}$ and the sequence $\left|\mathcal{D D P}_{n}^{*}\right|$ are as at the time of submitting this paper not in Sloane [18]. For some computed values of $F(n ; p)$, see Tables 3.1 and 3.2.

Table 3.1

\begin{tabular}{|c|c|c|c|c|c|c|c|c|c|}
\hline$n \backslash p$ & 0 & 1 & 2 & 3 & 4 & 5 & 6 & 7 & $\sum F(n ; p)=\left|\mathcal{D D P}_{n}^{*}\right|$ \\
\hline 0 & 1 & & & & & & & & 1 \\
\hline 1 & 1 & 1 & & & & & & & 2 \\
\hline 2 & 1 & 3 & 0 & & & & & & 4 \\
\hline 3 & 1 & 6 & 1 & 0 & & & & & 8 \\
\hline 4 & 1 & 10 & 3 & 0 & 0 & & & & 14 \\
\hline 5 & 1 & 15 & 7 & 1 & 0 & 0 & & & 24 \\
\hline 6 & 1 & 21 & 13 & 3 & 0 & 0 & 0 & & 38 \\
\hline 7 & 1 & 28 & 22 & 8 & 1 & 0 & 0 & 0 & 60 \\
\hline
\end{tabular}

Table 3.2

\begin{tabular}{|c|c|c|c|c|c|c|c|c|c|}
\hline$n \backslash p$ & 0 & 1 & 2 & 3 & 4 & 5 & 6 & 7 & $\sum F(n ; p)=\left|\mathcal{D D P}_{n}\right|$ \\
\hline 0 & 1 & & & & & & & & 1 \\
\hline 1 & 1 & 1 & & & & & & & 2 \\
\hline 2 & 1 & 3 & 1 & & & & & & 5 \\
\hline 3 & 1 & 6 & 5 & 1 & & & & & 13 \\
\hline 4 & 1 & 10 & 13 & 5 & 1 & & & & 30 \\
\hline 5 & 1 & 15 & 27 & 16 & 6 & 1 & & & 137 \\
\hline 6 & 1 & 21 & 48 & 38 & 21 & 7 & 1 & & 279 \\
\hline 7 & 1 & 28 & 78 & 78 & 57 & 28 & 8 & 1 & \\
\hline
\end{tabular}

\section{Number of $\mathcal{D}^{*}$-classes}

For the definitions of the Green's relations $(\mathcal{L}, \mathcal{R}$, and $\mathcal{D})$ and their starred analogues $\left(\mathcal{L}^{*}, \mathcal{R}^{*}\right.$, and $\left.\mathcal{D}^{*}\right)$, we refer the readers to Howie [12] and Fountain [8], (respectively) or Ganyushkin and Mazorchuk [9]. 
First, notice that from ([1], Lemma 2.1), we deduce that number of $\mathcal{L}$-classes in $K(n, p)=\left\{\alpha \in \mathcal{D P} \mathcal{P}_{n}: h(\alpha)=p\right\}$ (as well as the number of $\mathcal{R}$-classes there) is $\left(\begin{array}{l}n \\ p\end{array}\right)$. To describe the $\mathcal{D}$-classes in $\mathcal{D P}{ }_{n}$ and $\mathcal{O D P}{ }_{n}$, first we recall (from [1]) that the gap and reverse gap of the image set of $\alpha$ (with $h(\alpha)=p$ ) are ordered $(p-1)$-tuples defined as follows:

$$
g(\operatorname{Im} \alpha)=\left(\left|a_{2} \alpha-a_{1} \alpha\right|,\left|a_{3} \alpha-a_{2} \alpha\right|, \ldots,\left|a_{p} \alpha-a_{p-1} \alpha\right|\right),
$$

and

$$
\begin{gathered}
\left.g^{R}(\operatorname{Im} \alpha)=\left(\left|a_{p} \alpha-a_{p-1} \alpha\right|\right), \ldots,\left|a_{3} \alpha-a_{2} \alpha\right|,\left|a_{2} \alpha-a_{1} \alpha\right|\right), \\
\text { where } \alpha=\left(\begin{array}{cccc}
a_{1} & a_{2} & \ldots & a_{p} \\
a_{1} \alpha & a_{2} \alpha & \ldots & a_{p} \alpha
\end{array}\right) \text { with } 1 \leq a_{1}<a_{2}<\cdots<a_{p} \leq n .
\end{gathered}
$$

Further, let $d_{i}=\left|a_{i+1} \alpha-a_{i} \alpha\right|$ for $i=1,2, \ldots, p-1$. Then

$$
g(\operatorname{Im} \alpha)=\left(d_{1}, d_{2}, \ldots, d_{p-1}\right) \text { and } g^{R}(\operatorname{Im} \alpha)=\left(d_{p-1}, d_{p-2}, \ldots, d_{1}\right) .
$$

For example, if

$$
\alpha=\left(\begin{array}{lllll}
1 & 2 & 4 & 7 & 8 \\
3 & 4 & 6 & 9 & 10
\end{array}\right), \beta=\left(\begin{array}{cccc}
2 & 4 & 7 & 8 \\
10 & 8 & 5 & 4
\end{array}\right) \in \mathcal{D P}_{10},
$$

then $g(\operatorname{Im} \alpha)=(1,2,3,1), g(\operatorname{Im} \beta)=(2,3,1), g^{R}(\operatorname{Im} \alpha)=(1,3,2,1)$ and $g^{R}(\operatorname{Im} \beta)=(1,3,2)$. Next, let $d(n, p)$ be the number of distinct ordered p-tuples: $\left(d_{1}, d_{2}, \ldots, d_{p}\right)$ with $\sum_{i=1}^{p} d_{i}=n$. This is clearly the number of compositions of $n$ into $p$ parts. Thus, we have 
Lemma 3.1 ([17, p.151). $d(n, p)=\left(\begin{array}{c}n-1 \\ p-1\end{array}\right)$.

We shall henceforth use the following well-known binomial identity when needed:

$$
\sum_{m=p}^{n}\left(\begin{array}{l}
m \\
p
\end{array}\right)=\left(\begin{array}{c}
n+1 \\
p+1
\end{array}\right)
$$

We take this opportunity to state and prove a result which was omitted in [2].

Theorem 3.2. Let $S=\mathcal{O D P}_{n}$. Then

(1) the number of $\mathcal{D}$-classes in $K(n, p)(p \geq 1)$ is $\left(\begin{array}{l}n-1 \\ p-1\end{array}\right)$;

(2) the number of $\mathcal{D}$-classes in $S$ is $1+2^{n-1}$.

\section{Proof.}

(1) It follows from ([1], Theorem 2.5): $(\alpha, \beta) \in \mathcal{D}$ if and only if $g(\operatorname{Im} \alpha)$ $=g(\operatorname{Im} \beta)$; ([1], Lemma 3.3): $p-1 \leq \sum_{i=1}^{p-1} d_{i} \leq n-1$; Lemma 3.1; and so the number of $\mathcal{D}$-classes is $\sum_{i=p-1}^{n-1} d(i, p-1)=\sum_{i=p-1}^{n-1}\left(\begin{array}{c}i-1 \\ p-2\end{array}\right)=\left(\begin{array}{c}n-1 \\ p-1\end{array}\right)$.

(2) The number of $\mathcal{D}$-classes in $S$ is $1+\sum_{p=1}^{n}\left(\begin{array}{l}n-1 \\ p-1\end{array}\right)=1+2^{n-1}$.

The following results from [13] will be needed:

Lemma 3.3 ([13], Lemma 2.3). Let $\alpha, \beta \in \mathcal{D D P}_{n}$ or $\mathcal{O D D P}_{n}$. Then

(1) $\alpha \leq_{\mathcal{R}^{*}} \beta$ if and only if Dom $\alpha \subseteq \operatorname{Dom} \beta$;

(2) $\alpha \leq_{\mathcal{L}^{*}} \beta$ if and only if $\operatorname{Im} \alpha \subseteq \operatorname{Im} \beta$;

(3) $\alpha \leq_{\mathcal{H}^{*}} \beta$ if and only if $\operatorname{Dom} \alpha \subseteq \operatorname{Dom} \beta$ and $\operatorname{Im} \alpha \subseteq \operatorname{Im} \beta$. 
From ([13], (3)), for $\alpha, \beta \in \mathcal{D D P}_{n}$, we have $(\alpha, \beta) \in \mathcal{D}^{*}$ if and only if

$$
g(\operatorname{Im} \alpha)=\left\{\begin{array}{l}
g(\operatorname{Im} \beta) ; \text { or } \\
g^{R}(\operatorname{Im} \beta), \text { if } p \leq a_{p}-a_{1} \leq(n-1) / 2 .
\end{array}\right.
$$

Similarly, from ([13], (4)), for $\alpha, \beta \in \mathcal{O D D P}_{n}$, we have

$$
(\alpha, \beta) \in \mathcal{D}^{*} \text { if and only if } g(\operatorname{Im} \alpha)=g(\operatorname{Im} \beta) .
$$

Now a corollary of Theorem 3.2 follows:

Corollary 3.4. Let $S=\mathcal{O D D P}_{n}$. Then

(1) the number of $\mathcal{D}^{*}$-classes in $K(n, p)(p \geq 1)$ is $\left(\begin{array}{l}n-1 \\ p-1\end{array}\right)$;

(2) the number of $\mathcal{D}^{*}$-classes in $S$ is $1+2^{n-1}$.

Observe that for all $\alpha \in \mathcal{D P} \mathcal{P}_{n}$ with $h(\alpha)=p$,

$$
a_{p}-a_{1}=\sum_{i=1}^{p-1}\left(a_{i+1}-a_{i}\right)=\sum_{i=1}^{p-1} d_{i}
$$

where $g(\operatorname{Dom} \alpha)=\left(d_{1}, d_{2}, \ldots, d_{p-1}\right)$. Moreover, an ordered $p$-tuple: $\left(d_{1}, d_{2}, \ldots, d_{p}\right)$ is said to be symmetric if

$$
\left(d_{1}, d_{2}, \ldots, d_{p}\right)=\left(d_{1}, d_{2}, \ldots, d_{p}\right)^{R}=\left(d_{p}, d_{p-1}, \ldots, d_{1}\right) .
$$

Now, let $d_{s}(n, p)$ be the number of distinct symmetric ordered $p$-tuples: $\left(d_{1}, d_{2}, \ldots, d_{p}\right)$ with $\sum_{i=1}^{p} d_{i}=n$. Then we have

Lemma 3.5 ([2, Lemma 3.5).

$$
d_{s}(n ; p)= \begin{cases}0, & \text { if } n \text { is odd and } p \text { is even } \\ \left\lfloor\frac{n-1}{2}\right\rfloor \\ \left.\left\lfloor\frac{p-1}{2}\right\rfloor\right), & \text { otherwise. }\end{cases}
$$


Now by virtue of (5) and ([1], Theorem 2.5), it is not difficult to see that the number of $\mathcal{D}^{*}$-classes in $\mathcal{D D P} \mathcal{P}_{n}$ is the same as the number of $\mathcal{D}$-classes in $\mathcal{O D P}_{n}$ less those pairs that are merged into single $\mathcal{D}^{*}$-classes in $\mathcal{D D P}_{n}$. Thus, we have

Lemma 3.6. Let $g(m, p)$ be the number of $\mathcal{D}$-classes in $\mathcal{O D P}_{n}$ (consisting of maps of height $p$ and $\sum d_{i}=m$ ) that are merged into single $\mathcal{D}^{*}$-classes in $\mathcal{D D P}_{n}$. Then $m \leq(n-1) / 2$, and

$$
g(m, p)= \begin{cases}\frac{1}{2}\left(\begin{array}{l}
m-1 \\
p-2
\end{array}\right), & \text { if } n \text { is odd and } p \text { is odd } \\
\frac{1}{2}\left[\left(\begin{array}{l}
m-1 \\
p-2
\end{array}\right)-\left(\begin{array}{l}
\left\lfloor\frac{m-1}{2}\right\rfloor \\
\left\lfloor\frac{p-2}{2}\right\rfloor
\end{array}\right),\right. & \text { otherwise. }\end{cases}
$$

Proof. The result follows from (5), Lemmas $3.1 \& 3.5$ and the observation that

$$
g(n, p)=\frac{d(n-1, p-1)-d_{s}(n-1, p-1)}{2} .
$$

Now we have the main result of this section.

Theorem 3.7. Let $B(n, p)$ be the number of $\mathcal{D}$-classes in $\mathcal{O D P}_{n}$ (consisting of maps of height $p$ ) that are merged into single $\mathcal{D}^{*}$-classes in $\mathcal{D D P}_{n}$. Then for $n \geq p \geq 1$, we have

$$
B(n, p)= \begin{cases}\frac{1}{2}\left[\left(\begin{array}{l}
\left\lfloor\frac{n-1}{2}\right\rfloor \\
p-1
\end{array}\right)-\left(\begin{array}{c}
\left\lfloor\frac{n-1}{4}\right\rfloor \\
\frac{p-1}{2}
\end{array}\right),\right. & \text { if } p \text { is odd } ; \\
\frac{1}{2}\left[\left(\begin{array}{l}
\left\lfloor\frac{n-1}{2}\right\rfloor \\
p-1
\end{array}\right)-2\left(\begin{array}{c}
\left\lfloor\frac{n-1}{4}\right\rfloor \\
\frac{p}{2}
\end{array}\right)\right], & \text { if } n \equiv 1,2(\bmod 4), \& p \text { is even; } \\
\frac{1}{2}\left[\left(\begin{array}{l}
\left\lfloor\frac{n-1}{2}\right\rfloor \\
p-1
\end{array}\right)-2\left(\begin{array}{c}
\left\lfloor\frac{n-3}{4}\right\rfloor \\
\frac{p}{2}
\end{array}\right)-\left(\begin{array}{l}
\left\lfloor\frac{n-3}{4}\right\rfloor \\
\left.\frac{p-2}{2}\right)
\end{array}\right),\right. & \text { if } n \equiv-1,0(\bmod 4), \& p \text { is even. }\end{cases}
$$


Proof. The result follows from (5), (7) and Lemma 3.6. To see this, let $n \equiv 0(\bmod 4)$ and $p$ be even. Then $n=4 k$ for some integer $k$, and

$$
\begin{aligned}
& B(n, p)=\sum_{m=p}^{\left\lfloor\frac{n-1}{2}\right\rfloor} g(m, p)=\sum_{m=p}^{2 k-1} g(m, p) \\
& =g(p, p)+g(p+2, p)+\cdots+g(2 k-2, p) \\
& +g(p+1, p)+g(p+3, p)+\cdots+g(2 k-1, p) \\
& =\frac{1}{2}\left[\left(\begin{array}{l}
p-1 \\
p-2
\end{array}\right)-\left(\begin{array}{c}
\frac{p-2}{2} \\
\frac{p-2}{2}
\end{array}\right)+\left(\begin{array}{l}
p+1 \\
p-2
\end{array}\right)-\left(\begin{array}{c}
\frac{p}{2} \\
\frac{p-2}{2}
\end{array}\right)+\cdots+\left(\begin{array}{l}
2 k-3 \\
p-2
\end{array}\right)-\left(\begin{array}{c}
k-2 \\
\frac{p-2}{2}
\end{array}\right)\right] \\
& +\frac{1}{2}\left[\left(\begin{array}{c}
p \\
p-2
\end{array}\right)-\left(\begin{array}{c}
\frac{p}{2} \\
\frac{p-2}{2}
\end{array}\right)+\left(\begin{array}{c}
p+2 \\
p-2
\end{array}\right)-\left(\begin{array}{c}
\frac{p+2}{2} \\
\frac{p-2}{2}
\end{array}\right)+\cdots+\left(\begin{array}{c}
2 k-2 \\
p-2
\end{array}\right)-\left(\begin{array}{c}
k-1 \\
\frac{p-2}{2}
\end{array}\right)\right] \\
& =\frac{1}{2}\left[\left(\begin{array}{c}
2 k-1 \\
p-1
\end{array}\right)-2\left(\begin{array}{c}
k-1 \\
\frac{p}{2}
\end{array}\right)-\left(\begin{array}{c}
k-1 \\
\frac{p-2}{2}
\end{array}\right)\right] \\
& =\frac{1}{2}\left[\left(\begin{array}{l}
\frac{n-2}{2} \\
p-1
\end{array}\right)-2\left(\begin{array}{l}
\frac{n-4}{4} \\
\frac{p}{2}
\end{array}\right)-\left(\begin{array}{c}
\frac{n-4}{4} \\
\frac{p-2}{2}
\end{array}\right)\right] .
\end{aligned}
$$

All the other cases are handled similarly.

Now we have the main result of this section.

Corollary 3.8. The number of $\mathcal{D}^{*}$-classes in $\mathcal{D D P}_{n}$ (consisting of maps of height $p \geq 1)$ is $\left(\begin{array}{l}n-1 \\ p-1\end{array}\right)-B(n, p)$.

Proof. The result follows from Theorem 3.7 and the remarks preceding Lemma 3.6. 
Corollary 3.9. The number of $\mathcal{D}^{*}$-classes in $\mathcal{D D P}{ }_{n}$ denoted by $d_{n}$ is

$$
d_{n}= \begin{cases}2^{n-1}-2^{\left\lfloor\frac{n-3}{2}\right\rfloor}+\cdot 2^{\left\lfloor\frac{n+1}{4}\right\rfloor}, & \text { if } n \equiv-1,0(\bmod 4) \\ 2^{n-1}-2^{\left\lfloor\frac{n-3}{2}\right\rfloor}+3 \cdot 2^{\left\lfloor\frac{n-3}{4}\right\rfloor}, & \text { if } n \equiv 1,2(\bmod 4) .\end{cases}
$$

Proof. The result follows from Theorem 3.7 and Corollary 3.8. To see this, let $n \equiv 1,2(\bmod 4)$. Then $n=4 k+1,4 k+2$ for some integer $k$, and

$$
\begin{aligned}
& d_{n}=1+\sum_{p=1}^{n}\left(\begin{array}{l}
n-1 \\
p-1
\end{array}\right)-\sum_{p=1}^{\left\lfloor\frac{n-1}{2}\right\rfloor} B(n, p)=1+2^{n-1}-\sum_{p=1}^{2 k} B(n, p) \\
& =1+2^{n-1}-[B(n, 1)+B(n, 3)+\cdots+B(n, 2 k-1)] \\
& -[B(n, 2)+B(n, 4)+\cdots+B(n, 2 k)] \\
& =1+2^{n-1}-\frac{1}{2}\left[\left(\begin{array}{l}
2 k \\
0
\end{array}\right)-\left(\begin{array}{l}
k \\
0
\end{array}\right)+\left(\begin{array}{l}
2 k \\
2
\end{array}\right)-\left(\begin{array}{l}
k \\
1
\end{array}\right)+\cdots+\left(\begin{array}{c}
2 k \\
2 k-2
\end{array}\right)-\left(\begin{array}{c}
k \\
k-1
\end{array}\right)\right] \\
& -\frac{1}{2}\left[\left(\begin{array}{c}
2 k \\
1
\end{array}\right)-2\left(\begin{array}{l}
k \\
1
\end{array}\right)+\left(\begin{array}{l}
2 k \\
3
\end{array}\right)-2\left(\begin{array}{l}
k \\
2
\end{array}\right)+\cdots+\left(\begin{array}{c}
2 k \\
2 k-1
\end{array}\right)-2\left(\begin{array}{l}
k \\
k
\end{array}\right)\right] \\
& =1+2^{n-1}-\frac{1}{2}\left[\left(2^{2 k}-1\right)-\left(3 \cdot 2^{k}+1\right)+2\right] \\
& =2^{n-1}-2^{\left\lfloor\frac{n-3}{2}\right\rfloor}+3 \cdot 2^{\left\lfloor\frac{n-3}{4}\right\rfloor} \text {. }
\end{aligned}
$$

The case $n \equiv-1,0(\bmod 4)$ is handled similarly.

\section{Acknowledgements}

The second named author would like to thank Bowen University, Iwo and Sultan Qaboos University for their financial support and hospitality, respectively. 


\section{References}

[1] F. Al-Kharousi, R. Kehinde and A. Umar, On the semigroup of partial isometries of a finite chain, Comm. Algebra 44(2) (2016), 639-647.

DOI: https://doi.org/10.1080/00927872.2014.984838

[2] F. Al-Kharousi, R. Kehinde and A. Umar, Combinatorial results for certain semigroups of partial isometries of a finite chain, Australas. J. Combin. 58(3) (2014), 365-375.

[3] D. Borwein, S. Rankin and L. Renner, Enumeration of injective partial transformations, Discrete Math. 73(3) (1989), 291-296.

DOI: https://doi.org/10.1016/0012-365X(89)90272-0

[4] L. Bracci and L. E. Picasso, Representations of semigroups of partial isometries, Bull. Lond. Math. Soc. 39(5) (2007), 792-802.

DOI: https://doi.org/10.1112/blms/bdm059

[5] V. H. Fernandes, The monoid of all injective orientation preserving partial transformations on a finite chain, Comm. Algebra 28(7) (2000), 3401-3426.

DOI: https://doi.org/10.1080/00927870008827033

[6] V. H. Fernandes, G. M. S. Gomes and M. M. Jesus, The cardinal and the idempotent number of various monoids of transformations on a finite chain, Bull. Malays. Math. Sci. Soc. 34(1) (2011), 79-85.

[7] J. B. Fountain, Adequate semigroups, Proc. Edinburgh Math. Soc. 22(2) (1979), 113-125.

$$
\text { DOI: https://doi.org/10.1017/S0013091500016230 }
$$

[8] J. B. Fountain, Abundant semigroups, Proc. London Math. Soc. 44(1) (1982), 103-129.

$$
\text { DOI: https://doi.org/10.1112/plms/s3-44.1.103 }
$$

[9] O. Ganyushkin and V. Mazorchuk, Classical Finite Transformation Semigroups: An Introduction, Springer-Verlag, London, 2009.

[10] G. U. Garba, Nilpotents in semigroups of partial one-to-one order-preserving mappings, Semigroup Forum 48(1) (1994), 37-49.

DOI: https://doi.org/10.1007/BF02573652

[11] V. Gould, Graph expansions of right cancellative monoids, Internat. J. Algebra Comput. 6(6) (1996), 713-733.

DOI: https://doi.org/10.1142/S0218196796000404

[12] J. M. Howie, Fundamentals of Semigroup Theory, London Mathematical Society Monographs, New Series 12, Oxford Science Publications, The Clarendon Press, Oxford University Press, New York, 1995. 
[13] R. Kehinde, S. O. Makanjuola and A. Umar, On the semigroup of order-decreasing partial isometries of a finite chain (Submitted).

[14] A. Laradji and A. Umar, Combinatorial results for the symmetric inverse semigroup, Semigroup Forum 75(1) (2007), 221-236.

DOI: https://doi.org/10.1007/s00233-007-0732-8

[15] M. V. Lawson, Inverse semigroups, The theory of partial symmetries, World Scientific Publishing Co., Inc., River Edge, NJ, 1998.

[16] S. Lipscomb, Symmetric Inverse Semigroups, Mathematical Surveys and Monographs, 46, Amer. Math. Soc., Providence, R.I., 1996.

[17] J. Riordan, Combinatorial Identities, John Wiley and Sons, New York, 1968.

[18] N. J. A. Sloane, (Edition), The On-Line Encyclopedia of Integer Sequences, 2011.

Available at http://oeis.org/

[19] A. Umar, On the semigroups of partial one-to-one order-decreasing finite transformations, Proc. Roy. Soc. Edinburgh Sect. A: Math. 123(2) (1993), 355-363.

DOI: https://doi.org/10.1017/S0308210500025737

[20] A. Umar, Some combinatorial problems in the theory of symmetric inverse semigroups, Algebra Discrete Math. 9(2) (2010), 115-126.

[21] Lawrence J. Wallen, Semigroups of partial isometries, Bull. Amer. Math. Soc. 75(4) (1969), 763-764.

DOI: https://doi.org/10.1090/S0002-9904-1969-12278-0 